\title{
PROCESS AND SYSTEMS Barriers to staff reporting adverse incidents in NHS hospitals
}

\author{
Authors: Joanna Lucy Bovis, ${ }^{A}$ John Pradeep Edwin, ${ }^{B}$ Chris Patrick Bano, ${ }^{C}$ Athanasios Tyraskis, ${ }^{D}$ Dinnish Baskaran ${ }^{\mathrm{E}}$ \\ and Karthik Karuppaiah ${ }^{\mathrm{F}}$
}

Our study assessed barriers to reporting adverse incidents (AIs). Adverse incident reporting (AIR), although it is a pillar of risk management, has a wide variation in staff perception and usage. A questionnaire was used in five NHS hospitals to assess 267 members of multidisciplinary team (MDT) staff usage of AIR. Thirty-three percent of staff had never reported an adverse incident (AI). Fourty-one percent of staff had missed opportunities to report AIs due to a poor response to previous reports. The group who missed opportunities had a significantly higher proportion of not having received feedback to their previous AI ( $p=0.03)$. In the group who had received training, $79 \%$ had submitted an AI. This was significantly higher than the group who had not received training $(63 \%, p=0.02)$. Our study revealed that training and feedback following AIR are two major factors that could improve confidence in and use of AI reporting.

KEYWORDS: Patient safety, clinical governance, duty of candour, safety culture

\section{Introduction}

An adverse incident (AI) is an event that causes, or has the potential to cause, unexpected or unwanted effects that will involve the safety of patients, staff, users and other people. 1 The importance of an incident is not always obvious, and types of incidents can vary from the extremely serious (death or serious injury) to the apparently minor. Adverse incident reporting (AIR) is the principal way in which NHS staff can raise concerns about patient safety, risks and drive changes in the system. ${ }^{1}$

Adverse incident reporting is a cornerstone in clinical governance and it supports health service improvement by providing information, guidance and recommendations through which resources can be directed to areas where they are most needed

Authors: ${ }^{\text {A }}$ core surgical trainee, King's College Hospital, London, UK; ${ }^{B}$ orthopaedic specialty doctor, King's College Hospital, London, UK; ' Core surgical trainee, King's College Hospital, London,

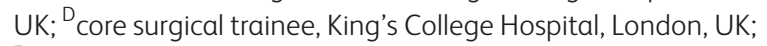

Eorthopaedic registrar, King's College Hospital, London, UK;

F consultant orthopaedic surgeon, King's College Hospital, London, UK to improve quality and safety. ${ }^{2}$ Whether it is with the purpose of whistle blowing or for duty of candour, there are multiple methods staff can use to escalate their concerns. Including reporting directly to their line manager or local 'freedom to speak up' guardian, there are multiple external agencies that can be contacted by staff directly such as the Care Quality Commission and NHS Improvement. Practically, an AI report is the most convenient way for staff within the NHS to start the process of raising a concern. Adverse incident reporting is intended to open up the circumstances surround the incident, correct mistakes, identify good medical practice and learn lessons for the future so as to prevent recurrence of similar incidents

The Department of Health (DH) recommends that all staff groups in the NHS should report all AIs, including near misses and minor safety issues as trending of these incidents can indicate serious underlying issues. ${ }^{3}$ Adverse incident reporting develops a culture in which system error and human error can be identified, openly reported, analysed, rectified and resolved. ${ }^{1-3}$

At the turn of the millennium, a government review found that the culture within the NHS was: 'Not one that encourages reporting and analysis (of adverse incidents)'. While there is a robust technical AI reporting infrastructure in place, underreporting remains, accompanied by a culture of defensiveness and blame. ${ }^{1}$ Our study was designed to evaluate the experiences of different healthcare staff in adverse incident reporting and to identify factors that influence reporting of AIs.

\section{Methods}

We conducted a cross-sectional staff survey with a questionnaire (Supplementary file 1) across five NHS Hospitals in the South East of England. The hospitals that were involved in the study include a major trauma centre (MTC), an elective orthopaedic unit and three district general hospitals (DGHs). From these hospitals, 267 staff members participated in the study (Fig 1). Staff members were asked by various members of the author team to complete a paper form during a 12-month period. Staff members were largely members of the MDT that the authors worked with during this time and were asked at random. The questionnaires were completed by various staff groups that included consultants (12\%), middle-grade doctors (34\%), foundation doctors (12\%), nursing staff of all levels $(30 \%)$ and allied healthcare professionals (12\%).

The questions were divided into three groups. The first one evaluated the staff members' awareness of AI systems, whether 
Fig 1. Staff surveyed. $A \& E=$ accident and emergency

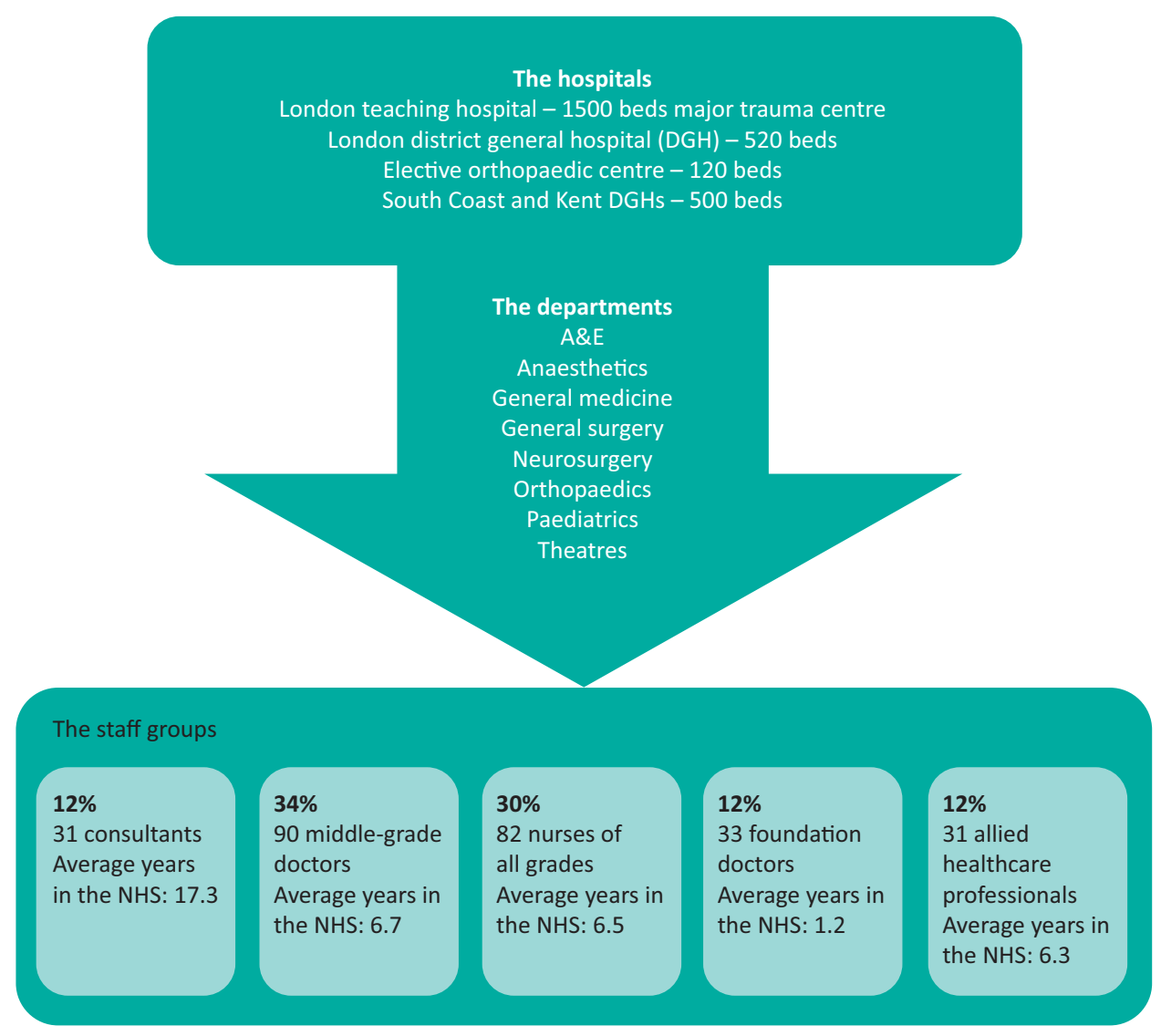

they had used an AI, experience of AI training, understanding of AIs and how they are handled. The second group of questions focused on the experience following reporting an AI, including whether or not the primary issue was addressed, whether the AI resulted in change in clinical practice and if there were subsequent similar adverse incidents noted. The third set of questions assessed the staff member's perspective on AI reporting, such as whether opportunities to submit subsequent AIRs were missed as a consequence of initial AI handling and whether there was awareness of how to follow up an AI report that didn't seem to have been acted on.

As a staff audit, not involving patients, this study did not require ethical approval. Consent was given by each member of staff surveyed, when filling out the questionnaire, to have the results published. As no patients were involved, no consenting of patients was required.

\section{Results}

Of the 267 staff members surveyed, 87 (33\%) reported never having completed an AI report. This equated to eight out of 30 consultants surveyed with a mean length of time in the NHS of 17.3 years, 21 foundation doctors (mean NHS employment 1.2 years), 34 middlegrade doctors (mean NHS employment 6.7), 12 nurses (mean NHS employment 6.5 years) and 12 allied healthcare professionals (mean employment in the NHS 6.3 years) (Table 1).

Table 1. Questionnaire results

\begin{tabular}{lllll} 
Staff group & $\begin{array}{l}\text { Has completed } \\
\text { an AI } \mathbf{n}(\%)\end{array}$ & $\begin{array}{l}\text { Has not completed } \\
\text { an AI } \mathbf{n}(\%)\end{array}$ & $\begin{array}{l}\text { Feels they understand } \\
\text { the AI system } \mathbf{n}(\%)\end{array}$ & $\begin{array}{l}\text { Has received training } \\
\text { in AI reporting } \mathbf{n}(\%)\end{array}$ \\
Consultants & $22(73)$ & $8(27)$ & $7(22)$ & $5(16)$ \\
Middle-grade doctors & $56(62)$ & $34(38)$ & $23(26)$ & $20(22)$ \\
Foundation doctors & $13(38)$ & $21(61)$ & $6(18)$ & $29(88)$ \\
Nurses & $70(85)$ & $12(15)$ & $19(23)$ & $30(36)$ \\
Allied healthcare professionals & $19(61)$ & $12(39)$ & $7(23)$ & $13(42)$ \\
All staff & $180(67)$ & $87(33)$ & $62(23)$ & $97(36)$ \\
\hline AI = adverse incident & & &
\end{tabular}


Table 2. Questionnaire results - experience of reporting an AI

\begin{tabular}{|c|c|c|c|}
\hline & Yes n (\%) & No $n(\%)$ & Did not respond \\
\hline Did you receive feedback on the action taken as a result of your submitted AIR? & $51(28.3)$ & $129(71.7)$ & 0 \\
\hline Do you feel that the primary issue, about which you have submitted an AIR, was dealt with? & $59(32.7)$ & $116(64.4)$ & 5 \\
\hline Did you see the expected change in practice as a result of submitting the AIR? & $43(23.8)$ & $129(71.7)$ & 8 \\
\hline Have you noticed repetition of the same incident that you submitted an AIR about? & $108(60.0)$ & $61(33.8)$ & 11 \\
\hline $\begin{array}{l}\text { Have you missed opportunities to submit subsequent AIRs due to the way previous ones } \\
\text { submitted have been handled? }\end{array}$ & $73(40.5)$ & $98(54.4)$ & 9 \\
\hline
\end{tabular}

$\mathrm{AI}=$ adverse incident; $\mathrm{AIR}=$ adverse incident report

The responses suggest that there is limited confidence and knowledge of the system. Only 36\% ( $n=97)$ of staff could recall receiving training and $23 \%(n=62)$ reported confidence knowing how the process of AIR works. Of those staff who had filed an AI, only $28 \%(n=51)$ had received feedback and just $33 \%(n=59)$ felt that the primary issue had been resolved. Sixty percent ( $n=108$ ) of staff had noticed repeated incidences similar to the original incident they filed an AI about (Table 2).

There was a white space question asking for any comments on AIR and approximately $25 \%$ of responders wrote a comment. These responses supported the numerical data. A representative sample of comments are included in box 1 .

Within the group who had received training, $79 \%(n=57)$ of staff had submitted an AI. This was significantly higher than in the group who had not received training, of which $63 \%(n=105)$ had submitted an AI ( $p=0.02)$. In the group who reported confidence in understanding the AI process, $77 \%$ ( $n=92)$ of staff had submitted an AI. This was significantly higher than the group who did not understand the process, of which $59 \%(n=70)$ had submitted an AI $(p=0.01)$.

Of those who had completed an AI report $(n=180), 41 \%(n=73)$ had missed opportunities to report subsequent AIs, due to a poor response from previous AI submissions. To examine the possible reason for this we compared the rate of feedback received in the group that had missed opportunities and those who had not. We included 171 responders to this analysis (nine further responders were excluded due to not having answered the missed opportunity question). Interestingly, we found that those who had a missed opportunity to report an AI had a significantly higher proportion of not having received feedback to their previous AI; $81 \%(n=59)$ vs $64 \%(n=63)$ in the group who did not miss an opportunity $(p=0.03)$.

There was significant variation between hospitals, with the lowest rate of staff having used an AIR reported at the elective orthopaedic centre ( $40 \%$ of staff compared with $78 \%$ at the

\section{Box 1. Free-text feedback}

'I get no feedback, no point, never acted upon.'

'Do they even get read?'

'I wanted to do one, no idea how to do it'

'The AI system will only be useful if investigated, appropriate action taken and feedback given to person reporting'

'How do you know if an AI has been reported already? Keen to have teaching on this'
DGHs and $70 \%$ at the MTC). No variation between department was observed.

\section{Discussion}

Mistakes are an inevitable part of medical practice; ${ }^{4} 1.4$ million AIs are reported each year in the UK, with $75 \%$ from the hospital care sector. ${ }^{5}$ The principal way to improve safety and good medical practice in the NHS is to create an open culture among staff members: 'in which errors or service failures can be admitted, reported and discussed without fear of reprisal'. ${ }^{3}$

Multiple studies have shown that under reporting of AIs is significant. ${ }^{1,2,5}$ A large American report concluded that six out of seven AIs go unreported. ${ }^{6}$ In the NHS, a recent case notes review revealed significant underreporting ${ }^{7}$ which was subsequently discussed at a House of Commons Health Select Committee meeting. ${ }^{8}$

Despite recognition of opportunistic learning and reflection from existing reporting systems, there is a culture of underreporting, ${ }^{5}$ which may be attributable to multiple factors, as highlighted in the Francis Enquiry. These consist of target-driven priorities, low morale, disengagement from management, acceptance of poor standards and denial. ${ }^{9}$

The current study shows a significant proportion of staff members have not reported an AI despite working in the NHS for a collective 1900 years; here we try to examine the reasons for the underreporting.

Nursing staff were found to have more AIR experience compared to doctors. This is a trend reflected in other studies. ${ }^{10,11}$ Kingston et al attributed these discernible differences to habitual practice and cultural discrepancies where doctors view 'whistleblowing' as disloyal, unethical and unsupportive, while nursing staff are protocol driven and protective to minimise punitive repercussions. ${ }^{10}$

Our study also suggested that healthcare staff do not experience or view AIRs as effective clinical tools to improve patient safety as $64 \%$ of respondents did not think their primary concern had been addressed and $76 \%$ noted repeated incidences of the adverse incident after they had submitted an AIR. Overall, $44 \%$ of all healthcare staff had missed opportunities to file subsequent incident reports. This disengagement from AIR may be the result of initial AIR handling. Macrae suggested that AIR often remains a passive process of information transfer rather than participative improvement where staff can perceive AIR as a logging process awaiting solutions. ${ }^{12}$ There are 1.4 million AIR submitted in the NHS each year. ${ }^{5}$ If, as this study suggests, $44 \%$ of those that submit an AIR fail to submit a subsequent report, this means that over half a million incidents are not being reported on each year. 
The literature suggests that a significant proportion of patient safety reporting systems provide little feedback to staff. ${ }^{13,14}$ In an AIR questionnaire by Evans et al sent to 263 doctors and 799 nurses working in Australian hospitals, $57.7 \%$ of doctors and $61.8 \%$ of nurses reported a lack of feedback as a major barrier in AI reporting, demonstrating the importance of feedback in future AIR system engagement. ${ }^{10}$ Feedback has also been shown to be a critical determinant of success in improving AIR systems as it facilitates timely action of reports, reassures reporters that their concerns are acted upon ${ }^{15}$ and promotes AI reporting system compliance. $^{14}$

Our study highlighted a high level of underreporting among healthcare workers within the NHS across different healthcare settings. Experiences of initial AI handling have been widely been perceived negatively leading to disengagement from subsequent AI reporting. Reported feedback and training is low, but in institutions where higher feedback and training occurs, an associated increased AIR system compliance was present. Consequently, we recommend that all healthcare staff should receive adequate, standardised AIR training and robust feedback systems should be in place to promote a positive feedback reporting culture where patient safety can excel.

\section{Key learning points}

> AI reporting is the backbone for any healthcare system including NHS to drive good medical practice and to increase patient safety.

$>33 \%$ of health care professionals with a mean employment period of 7.0 years have never filled an AI.

$>71 \%$ of the staff members received no feedback following AIR and did not see any change in practice, of which $60 \%$ respondents had noticed repeated similar incidences and $41 \%$ missed further opportunities to report an AI.

$>$ Our study identified that lack of training and low levels of feedback are two major factors that lead to missed opportunities to submit AI reports.

\section{Author contributions}

JLB contributed to the data collection tool, data collection, coordination and write up. JE contributed to data collection. CPB contributed to data collection and editing the manuscript. AT contributed to statistical analysis. DB contributed to data analysis and writing of the manuscript. KK oversaw the project, coordinated data collection and edited the manuscript.

\section{Supplementary material}

Additional supplementary material may be found in the online version of this article at http://futurehospital.rcpjournal.org: S1 - Adverse incident report staff perceptions audit questionnaire

\section{References}

1 Department of Health. An organisation with a memory: report of an expert group on learning from adverse events in the NHS chaired by the Chief Medical Officer. London: DH, 2000.

2 Department of Health. Building a safer NHS for patients implementing an organisation with a memory. London: DH, 2001. http://webarchive.nationalarchives.gov.uk/20130107105354/ http://www.dh.gov.uk/en/Publicationsandstatistics/Publications/ PublicationsPolicyAndGuidance/Browsable/DH_4916275 [Accessed 19 February 2017].

3 Barach P, Small S. Reporting and preventing medical mishaps: lessons from non-medical near miss reporting systems. BMJ 2000;320:759-63.

4 Committee on quality of health care in America. To Err Is Human: Building a safer health system. National Academy of Sciences, 2000.

5 House of Commons. Public Administration Select Committee, 6th report 2014-15. Investigating clinical incidents in the NHS https://publications.parliament.uk/pa/cm201415/cmselect/ cmpubadm/886/886.pdf [Accessed 8.3.17].

6 Levinson DR. Adverse events in hospitals: national incidence among Medicare beneficiaries. Department of Health and Human Services, 2010. http://oig.hhs.gov/oei/reports/oei-06-09-00090.pdf. [Accessed 06 January 2017].

7 Sari AB, Sheldon TA, Cracknell A, Turnbull A. Sensitivity of routine system for reporting patient safety incidents in an NHS hospital: retrospective patient case note review, BMJ 2007;334:79.

8 House of Commons Select Committee for Health, Patient Safety, Sixth Report of Session 2008-9, Vol 1. https://publications. parliament.uk/pa/cm200809/cmselect/cmhealth/151/151i.pdf [Accessed 02 January 2017].

9 Francis R. The Mid Staffordshire NHS Foundation Trust Public Enquiry Final Report, 2013. http://webarchive.nationalarchives. gov.uk/20150407084231/http://www.midstaffspublicinquiry.com/ report [Accessed 08 March 2017].

10 Kingston MJ, Evans SM, Smith B], Berry JG. Attitudes of doctors and nurses towards incident reporting: a qualitative analysis. Med J Aust 2004;181:36-9.

11 Evans SM, Berry JG, Smith BJ et al. Attitudes and barriers to incident reporting: a collaborative hospital study. Qual Saf Health Care 2006;15:39-43

12 Macrae C. The problem with incident reporting, BMJ Qual Saf 2016;25:71-5.

13 Farley DO, Haviland A, Champagne S et al. Adverse-event-reporting practices by US hospitals: results of a national survey. Qual Saf Health Care 2008;17:416-23.

14 Benn J, Koutantji M, Wallace L et al. Feedback from incident reporting: information and action to improve patient safety. Qual Saf Health Care 2009;18:11-21.

Address for correspondence: Mr Karthik Karuppaiah, Orthopaedic Department, 2nd Floor, Hambleden Wing, King's College Hospital, Denmark Hill, London SE5 9RS, UK. Email: karthikkaruppaiah@nhs.net 